\title{
Rogue Waves and Mode Locking Driven by Vector Resonance Multimode Instability
}

\author{
Sergey V. Sergeyev", Hani Kbashi, and Stanislav A. Kolpakov \\ Aston Institute of Photonic Technologies, Aston University, Aston Triangle, Birmingham, B4 7ET, UK \\ ${ }^{*}$ Tel: +44(0)1212043504,e-mail: s.sergeyev@aston.ac.uk
}

\begin{abstract}
Modulation instabilities discovered more than fifty and hundred fifty years ago created since then a framework for study complexity of different wave phenomena including turbulence and rogue waves. Using Erbium-doped fiber laser without any previously studied mode-locking mechanisms, here for the first time we demonstrate both experimentally and theoretically a new type of modulation instability, namely Vector Resonance Multimode Instability, leading to tunability of the laser dynamics from turbulence including rogue waves to the stable pulse train similar to the laser mode-locking regime.
\end{abstract}

Keywords: fiber laser, rogue waves, polarization dynamics.

\section{INTRODUCTION}

Modulation instability (MI) was studied for the last fifty years to reveal emergence of spatial and temporal patterns in different systems including fluids, granular media, plasma, nonlinear optics and lasers [1]-[3]. For example, multimode Risken-Nummedal-Graham-Haken (RNGH) instability leads to existing the second lasing threshold exceeding of which results in excitation of a large number of the longitudinal spatial modes and generation of the pulse train with the period equals to the photon round-trip time. The history of the RNGH instability started in 1968 and since the extensive theoretical and experimental study of Er-doped fiber lasers revealed decreased second lasing threshold to the values slightly exceeding the value for the first threshold [2]. Here, we demonstrate experimentally and theoretically a new type of MI, namely Vector Resonance Multimode Instability (VRMI), that is behind complex behavior ranging from the turbulent including different types of rogue waves (breathers, twins, three sisters, crosses and accelerating) to the stable pulse train similar to the laser mode-locking regime $[4,5]$. We have justified experimentally and theoretically that increased in-cavity birefringence strength causes spatial modulation of the SOP of the in-cavity lasing field (with a period related to the beat length) and so leads through dispersion relation to the emergence of the additional satellite frequencies with the frequency splitting proportional to the birefringence strength. When the splitting is approaching the fundamental frequency inversely proportional to the photon round trip time, parametric resonance results in longitudinal modes synchronization and emergence of the pulse train with the narrow pulse width.

\section{EXPERIMENTAL STUDY}

The schematic of the unidirectional cavity fiber laser is shown in Fig. 1a. The cavity comprises of $1 \mathrm{~m}$ of Er-doped fiber (Liekki Er80-8/125) and $614 \mathrm{~m}$ of single mode fiber SMF-28. Optical isolator (OISO) with $51 \mathrm{~dB}$ back losses has been used to provide unidirectional lasing. The 80/20 fiber coupler was used to redirect the part of the signal outside the cavity. The cavity was pumped via a 1480/1550 WDM by using a $1480 \mathrm{~nm}$ laser diode (FOL14xx series) with an in-built isolator. The pump power was measured after the pump polarization controller (POC1) and the wavelength division multiplexing (WDM). The signal was detected by using a photodetector with a bandwidth of $17 \mathrm{GHz}$ (InGaAsUDP-15-IR-2 FC) connected to a $2.5 \mathrm{GHz}$ sampling oscilloscope (Tektronix DPO7254) and electrical spectrum analyzer (ROHDE and SCHWARZ FSV Signal Analyzer, $10 \mathrm{~Hz} / 13.6 \mathrm{GHz})$. The first lasing threshold for the continuous wave $(\mathrm{CW})$ regime was found in terms of the pump power as $16 \mathrm{~mW}$ whereas the second threshold of the multimode instability was of $18 \mathrm{~mW}$. In the experiments, we use special laser configuration and pump power of about hundred times less to exclude mode locking based on nonlinear polarization rotation [6]. The orientations of the paddles of the polarization controllers (POC1 and POC2) was set with respect to the vertical position at angles $\theta_{1}$ and $\theta_{2}$, namely $\theta_{1}=-59^{\circ}$ and $\theta_{2}=-78^{\circ},-74^{\circ},-69^{\circ}$. To characterize the resonance type of mode locking at the fast time scale we use a fast detector, oscilloscope, and electrical spectrum analyzer. Though the fundamental frequency was of $325.2 \mathrm{kHz}$, the radiofrequency (RF) spectrum was resolved much better in the case of $1000^{\text {th }}$ harmonic, i.e. around frequency of about 325.2 MHz. The RF spectrum shown in Fig. 1b reveals three types of peaks: the central peak of the $1000^{\text {th }}$ harmonic of the fundamental frequency, the two satellites tunable with the help of the POC2 and two closely located to the fundamental frequency satellites independent of POCs adjustment. It has been recently found that off-resonance conditions for the main peak and adjustable by POC2 satellites result in emergence rogue waves taking the form of chaotic oscillations similar to the shown in Fig. 1c for $\theta_{2}=-78^{\circ}$ [4]. As follows from the Fig. $1 \mathrm{a}-1 \mathrm{c}$, the POC2 adjustment towards resonance results in the regime stabilization similar to mode locking for the case when satellites match the main line (Fig. 1b). Unlike scalar multimode instability demonstrating oscillations close the harmonic with the period of the photon round-trip time $\tau_{\mathrm{R}}$ [2], the pulse train 
in Fig. 1c has the pulse width of $40 \mathrm{~ns}$ which is much less than the round trip time of $3 \mu \mathrm{s}$. This indicates the excitation of many longitudinal modes with their relative phases synchronized.
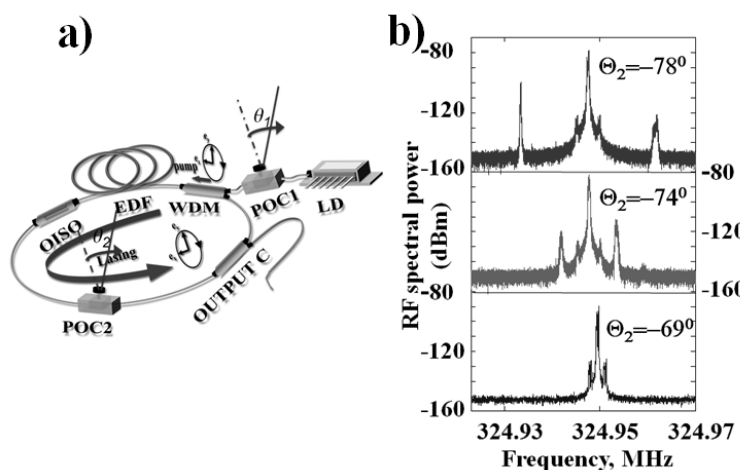

c)

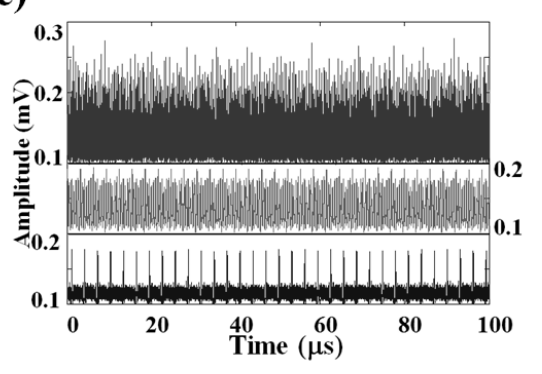

d)

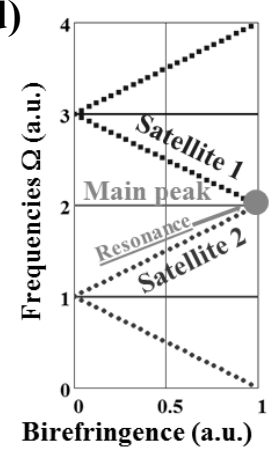

Figure 1: (a) Erbium doped fiber laser; (b) The RF spectrum and (c) corresponding oscillograms for different setting of the POC2: $\theta_{2}=-78^{\circ}, \theta_{2}=-74^{\circ}, \theta_{2}=-69^{\circ}$; d) Theoretical results demonstrating resonance between satellites and the main peak with adjustment of the in-cavity birefringence.

\section{THEORETICAL STUDY}

The experimental results can be well understood based on the vector model of an Er-doped fiber laser. The following rate equations are derived from the model suggested by Sergeyev et al. [5]:

$$
\begin{aligned}
& \frac{\partial S_{0}}{\partial z}+\frac{\partial S_{0}}{\partial t}=\left(\frac{2 \alpha_{1} f_{1}}{1+\Delta^{2}}-2 \alpha_{2}\right) S_{0}+\frac{2 \alpha_{1} f_{2}}{1+\Delta^{2}} S_{1}+\frac{2 \alpha_{1} f_{3}}{1+\Delta^{2}} S_{2}, \\
& \frac{\partial S_{1}}{\partial z}+\frac{\partial S_{1}}{\partial t}=\gamma S_{2} S_{3}+\left(\frac{2 \alpha_{1} f_{1}}{1+\Delta^{2}}-2 \alpha_{2}\right) S_{1}+\frac{2 \alpha_{1} f_{2}}{1+\Delta^{2}} S_{0}-\frac{2 \alpha_{1} f_{3} \Delta}{1+\Delta^{2}} S_{3}, \\
& \frac{\partial S_{2}}{\partial z}+\frac{\partial S_{2}}{\partial t}=-\gamma S_{1} S_{3}+\frac{2 \alpha_{1} f_{3}}{1+\Delta^{2}} S_{0}+\left(\frac{2 \alpha_{1} f_{1}}{1+\Delta^{2}}-2 \alpha_{2}\right) S_{2}+\left(\frac{2 \alpha_{1} f_{2} \Delta}{1+\Delta^{2}}-2 \beta\right) S_{3}, \\
& \frac{\partial S_{3}}{\partial z}+\frac{\partial S_{3}}{\partial t}=\frac{2 \alpha_{1} \Delta f_{3}}{1+\Delta^{2}} S_{1}-\frac{2 \alpha_{1} \Delta f_{2}}{1+\Delta^{2}} S_{2}+2 \beta S_{2}+\left(\frac{2 \alpha_{1} f_{1}}{1+\Delta^{2}}-2 \alpha_{2}\right) S_{3}, \\
& \frac{d f_{1}}{d t}=\varepsilon\left[\frac{\left(\chi_{s}-1\right) I_{p}}{2}-1-\left(1+\frac{I_{p} \chi_{p}}{2}+d_{1} S_{0}\right) f_{1}-\left(d_{1} S_{1}+\frac{I_{p}}{2} \xi\right) f_{2}-d_{1} S_{2} f_{3}\right], \\
& \frac{d f_{2}}{d t}=\varepsilon\left[\xi \frac{I_{p}\left(\chi_{s}-1\right)}{4}-\left(\frac{I_{p} \chi_{p}}{2}+1+d_{1} S_{0}\right) f_{2}-\left(\frac{I_{p} \chi_{p}}{2} \xi+d_{1} S_{1}\right) \frac{f_{1}}{2}\right], \\
& \frac{d f_{3}}{d t}=-\varepsilon\left[\frac{d_{1} S_{2} f_{1}}{2}+\left(\frac{I_{p} \chi_{p}}{2}+1+d_{1} S_{0}\right) f_{3}\right] .
\end{aligned}
$$

Here time is normalized to the round trip and length - to the cavity length; $S_{i}(i=0,1,2,3)$ are the Stokes parameters $\left(S_{0}\right.$ is the output power, pump and lasing powers are normalized to the corresponding saturation powers: $\left.S_{0}^{2}=S_{1}^{2}+S_{2}^{2}+S_{3}^{2}\right) ; \alpha_{1}$ is the total absorption of erbium ions at the lasing wavelength, $\alpha_{2}$ is the total insertion losses in cavity, $\beta$ is the birefringence strength $\left(2 \beta=2 \pi L / L_{\mathrm{b}}, L_{\mathrm{b}}\right.$ is the beat length); $\xi=\left(1-\delta^{2}\right) /\left(1+\delta^{2}\right)$ is parameter of the pump anisotropy, $\delta$ is the ellipticity of the pump wave, $\varepsilon=\tau_{\mathrm{R}} / \tau_{\mathrm{Er}}$ is the ratio of the round trip time $\tau_{\mathrm{R}}$ to the lifetime of erbium ions at the first excited level $\tau_{\mathrm{Er}} ; \chi_{\mathrm{p}, \mathrm{s}}=\left(\sigma_{\mathrm{a}}{ }^{(\mathrm{s}, \mathrm{p})}+\sigma_{\mathrm{e}}{ }^{(\mathrm{s}, \mathrm{p})}\right) / \sigma_{\mathrm{a}}{ }^{(\mathrm{s}, \mathrm{p})},\left(\sigma_{\mathrm{a}}{ }^{(\mathrm{s}, \mathrm{p})}\right.$ and $\sigma_{\mathrm{e}}{ }^{(\mathrm{s}, \mathrm{p})}$ are absorption and emission cross sections at the lasing (s) and pump (p) wavelengths); $\Delta$ is the detuning of the lasing wavelength with respect to the maximum of the gain spectrum (normalized to the gain spectral width; $d_{1}=\chi / \pi\left(1+\Delta^{2}\right)$; functions $f_{i}(i=1,2,3)$ are related to the angular distribution of the excited ions $n(\theta)$ expanded into a Fourier series as follows [5]:

$$
n(\theta)=\frac{n_{0}}{2}+\sum_{k=1}^{\infty} n_{1 k} \cos (k \theta)+\sum_{k=1}^{\infty} n_{2 k} \sin (k \theta), f_{1}=\left(\chi \frac{n_{0}}{2}-1\right)+\chi \frac{n_{12}}{2}, f_{2}=\left(\chi \frac{n_{0}}{2}-1\right)-\chi \frac{n_{12}}{2}, f_{3}=\chi \frac{n_{22}}{2} .
$$

Equations (1) have been derived under the approximation that the dipole moments of the absorption and emission transitions for erbium doped silica are located in the plane orthogonal to the direction of the light propagation [5]. Justification of such approximation is found in [7, 8]. As follows from the structure of Eqs. (1), birefringence in the laser cavity comprises two parts, viz. birefringence of the passive fiber combined with 
birefringence induced by the in-cavity polarization controller $2 \beta=2 \pi L / L_{b}$ and birefringence caused by polarization hole burning in the active fiber $\Delta \beta=2 \alpha_{1} f_{2} \Delta /\left(1+\Delta^{2}\right)$.

To specify conditions for the vector resonance mode locking, we linearize the Eqs. (1) in the vicinity of the

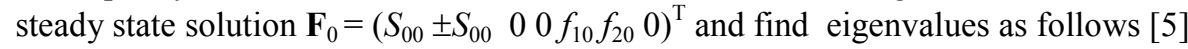

(I) $\lambda_{0}=i q+A_{0}\left(I_{p}, \xi\right)$,

(II) $\lambda_{1}=A_{1}\left(q, I_{p}, \xi\right)+i \Omega_{1}\left(q, I_{p}, \xi\right), \quad \lambda_{2}=A_{2}\left(q, I_{p}, \xi\right)+i \Omega_{2}\left(q, I_{p}, \xi\right), \lambda_{3}=A_{3}\left(q, I_{p}, \xi\right)+i \Omega_{3}\left(q, I_{p}, \xi\right)$,

(III) $\lambda_{4}=A_{4}\left(q, I_{p}, \beta, \xi\right)+i \Omega_{4}\left(q, I_{p}, \beta, \zeta\right), \lambda_{5}=A_{5}\left(q, I_{p}, \beta, \xi\right)+i\left(q+\Delta \Omega\left(q, I_{p}, \beta, \xi\right)\right)$,

$\lambda_{6}=A_{6}\left(q, I_{p}, \beta, \xi\right)+i\left(q-\Delta \Omega\left(q, I_{p}, \beta, \xi\right)\right)$,

$A_{0}\left(I_{p}, \xi\right)>0, A_{1}\left(q, I_{p}, \xi\right)<0, A_{2}\left(q, I_{p}, \xi\right)<0, A_{3}\left(q, I_{p}, \xi\right)<0, A_{4}\left(q, I_{p}, \xi\right)<0, A_{5}\left(q, I_{p}, \xi\right)>0, A_{6}\left(q, I_{p}, \xi\right)>0$.

Here $q=0, \pm 1, \pm 2, . . \pm N$ is the wave number of the longitudinal mode. All eigenvalues are normalized to the fundamental frequency $\omega=2 \pi / \tau_{\mathrm{R}}$. The obtained results for frequencies of branches (I) and (III) are shown in Fig. 1d. As follows from [5], the scalar branch II provides additional birefringence-independent satellites with the frequency splitting of $0.01 f$ ( $f$ is the fundamental frequency) with respect to the longitudinal mode frequency $q$. Though real part of the corresponding eigenvalues, i.e. $A_{1}, A_{2}$, and $A_{3}$, are less than zero, the satellites can be excited through parametric phase locking with the frequencies of the branch I [5]. As follows from Fig. 1d, increased birefringence strength can result in the resonance conditions where satellites frequencies for the longitudinal mode $q$ (branch III) matches the frequency of the longitudinal mode $q+N$ (where $N$ is integer) from the branch I. Thus, the theoretical results shown in Fig. 1d are in a good correspondence with the experimental results shown in Fig. 1b, i.e. matching the longitudinal modes' frequencies with the birefringence-dependent satellites results in their synchronization similar to the mode locking scenario through the injection locking [5].

\section{CONCLUSIONS}

In conclusion, we demonstrate experimentally and theoretically a new Vector Resonance Multimode Instability in erbium-doped fiber laser. By adjusting the in-cavity and the pump polarization controllers, we were able to tune the laser dynamics from the complex dynamics including rogue waves to the periodic similar to the mode locked regime. The increased birefringence strength leads to generation of satellite lines from the $q$-harmonic frequency that in the case of resonance with the $q+N$-harmonic, i.e. case when $N$-fold beat length equals to the cavity length, results in longitudinal modes synchronization. This synchronization is justified by decreased pulse width of $40 \mathrm{~ns}$ that much less than the round trip time of $3 \mu \mathrm{s}$. The demonstrated VRMI can be observed in many distributed systems where the spatial modulation of the system's variables can lead to the spectral splitting. The resonance of the split satellites with the other branch of eigen-frequencies can lead to the synchronization phenomena which can be of interest in photonics and beyond.

\section{ACKNOWLEDGEMENTS}

We acknowledge support from the Leverhulme Trust (Grant ref: RPG-2014-304).

\section{REFERENCES}

[1] G. P. Agrawal: Nonlinear Fiber Optics, San Diego: Academic Press, 2013.

[2] L. Lugiato, F. Prati, and M. Brambila, Nonlinear Optical Systems, Cambridge: Cambridge University Press, 2015.

[3] M. Onorato et al.: Rogue waves and their generating mechanism in different physical context. Phys. Rep. vol. 54, pp. 47-8, Jul. 2013.

[4] S. A. Kolpakov, H. Kbashi, and S. V. Sergeyev: Dynamics of vector rogue waves in a fiber laser with a ring cavity, Optica, vol. 3 , pp. 870-875, Aug. 2016.

[5] S. V. Sergeyev, H. Kbashi, N. Tarasov, Y. Loiko, and S. A. Kolpakov: Vector-resonance-multimode instability, Phys. Rev. Lett. , vol. 118, p. 033904, Jan. 2017.

[6] C. Lecaplain, Ph. Grelu, J. M. Soto-Crespo, and N. Akhmediev: Dissipative rogue waves generated by chaotic pulse bunching in a mode-locked laser, Phys. Rev. Lett., vol. 108, pp. 233901, Jun. 2012.

[7] R. Leners and G. Stéphan: Rate equation analysis of a multimode bipolarization $\mathrm{Nd}^{3+}$ doped fibre laser, Quantum Semiclass. Opt., vol. 7, pp. 757-794, Oct. 1995.

[8] S. V. Sergeyev: Spontaneous light-polarization symmetry breaking for an anisotropic ring-cavity dye laser, Phys. Rev. A, vol. 59, pp. 3909-3917, May 1999. 